\title{
Targeting Tumor Metabolism in Anti-Cancer Drug Discovery
}

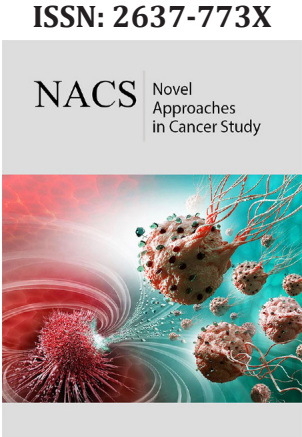

*Corresponding author: Alok Bhushan, Department of Pharmaceutical Sciences, Jefferson College of Pharmacy, Thomas Jefferson University + Philadelphia University, Philadelphia, PA 19107, USA

Submission: 栦 December 20, 2019

Published: 眥January 10, 2020

Volume 4 - Issue 1

How to cite this article: Bhushan A, Bhardwaj V \& Lai JCK (2020) Targeting Tumor Metabolism in Anti-Cancer Drug Discovery. Nov Appro in Can Study 4(1):318-330. NACS.000578.2020. DOI: 10.31031/NACS.2020.04.000578

Copyright@ Alok Bhushan, This article is distributed under the terms of the Creative Commons Attribution 4.0 International License, which permits unrestricted use and redistribution provided that the original author and source are credited.

\author{
Alok Bhushan ${ }^{1 *}$, Vikas Bhardwaj ${ }^{1}$ and James CK Lai ${ }^{2}$
}

${ }^{1}$ Department of Pharmaceutical Sciences, Jefferson College of Pharmacy, Thomas Jefferson University + Philadelphia University, Philadelphia, PA 19107, USA

${ }^{2}$ Department of Biomedical \& Pharmaceutical Sciences, College of Pharmacy, Division of Health Sciences, Idaho State University, Pocatello, ID 83209, USA

\begin{abstract}
Cancer cells have evolved to develop sets of survival strategies to enable them not only to survive and ward off apoptosis-inducing effects of most chemotherapeutic drugs in current use but also proliferate and invade their surrounding healthy tissue. In the 1920's, based on his pioneering research, Warburg hypothesized cancer cells rely on glycolysis for energy production to sustain their growth because their mitochondrial metabolism is dysfunctional. This review focuses on the current advances in cancer cell metabolism as a result of the recent resurgence of interests in the "Warburg hypothesis" (also called "Warburg effect") and discusses how these advances have revealed potential anti-cancer drug targets. Additionally, we will also discuss metabolic pathways that are critically coupled to cancer cell survival and proliferation, thereby uncovering other putative anti-cancer drug targets for therapeutic consideration. Thus, we hope to provide a forward-looking framework for discussing and designing new anti-cancer therapies.
\end{abstract}

Keywords: Targeting tumor metabolism; Tumor cell metabolic phenotypes; Warburg hypothesis or Effect; Reverse Warburg Effect; and Multi-target approach; Anti-cancer drug discovery

\section{Introduction}

Tumor cells have evolved to develop distinct sets of strategies to enable them not only to survive and ward off apoptosis-inducing effects of most chemotherapeutic drugs in current use, but also to proliferate and invade their surrounding healthy tissue. Most if not all of such adaptive strategies of tumor cells involve significant alterations of their metabolic phenotypes that differ markedly from those of normal, non-cancerous cells. Consequently, a critical assessment of how cancer cells modify their metabolism may reveal potential drug targets that allow the discovery and design of new and/or improved chemotherapeutic agents that will "kill" tumor cells. This review therefore focuses on highlighting the current advances as a result of the recent resurgence of interests in the "Warburg hypothesis" (also called "Warburg effect") and discusses how these advances have revealed potential anti-cancer drug targets. In parallel, we will discuss metabolic pathways that are critically coupled to tumor cell survival and proliferation, thereby uncovering other putative anti-cancer drug targets for therapeutic consideration. Finally, we will discuss several novel but practicable trends of research that will facilitate targeting tumor metabolism for anti-cancer drug discovery to follow the dictum "from bench to the bedside."

\section{The Warburg Hypothesis: Current Advances and Limitations}

\section{The Warburg hypothesis and its renaissance}

Historically, the "Warburg Hypothesis" could be traced back to the systematic investigation initiated by Otto Warburg and his co-workers commencing in the 1920's focusing on characterizing the metabolic phenotypes of multiple types of cancer cells $[1,2]$. Their pioneering work led to their observation that cancer cells appear to rely on glycolysis for energy production and survival despite the abundant availability of oxygen $[1,2]$. Those early discoveries prompted Warburg to hypothesize that cancer cells depend on glycolysis for energy supply because their mitochondrial oxidative metabolism is dysfunctional [2]. Also known as 'the Warburg effect' [3], the Warburg hypothesis has been neglected for almost a century until the recent resurgence of interests in "the role of metabolic programming in cancer progression" [4]. 
The "renaissance of the Warburg hypothesis" has given rise to a variety of studies aimed at elucidating the metabolic bases of the aggressive nature of many malignant cancers and their purported reliance on glycolysis for energy, survival, and proliferation [3-8]. While the initial phase of such studies seeks to uncover mechanisms that could account for the Warburg hypothesis, more recent studies either point to the limitations of some of the mechanistic explanations for the hypothesis or identify new facets of tumor metabolism that potentially could lead to the discovery of novel anti-tumor drug targets.

\section{Current advances and limitations}

The renewed interests in the Warburg hypothesis have motivated investigators to identify mechanistic explanations for the two key issues of the hypothesis:

(i) dysfunctional mitochondrial oxidative metabolism and

(ii) adaptation to hypoxia and shift to a glycolytic phenotype (i.e., the "glycolytic shift"). While those studies have undoubtedly enhanced our understanding of tumor metabolism, investigators have not been able to point out the limitations of such advances (see below).

\section{Mechanistic justification to explain:}

(i) Dysfunctional mitochondrial oxidative metabolism in cancers came from studies on mitochondrial genetics in cancers demonstrating that several mutations in mitochondrial DNA (mtDNA) occur in various cancer types [9]. Although the presence of mtDNA mutations in cancers can provide a mechanistic connection to the dysfunctional mitochondrial oxidative metabolism [9], recent evidence raises some doubt as to the validity of this mechanistic link, which merits a more critical reappraisal $[8,10]$. On the other hand, accumulating evidence suggest that loss-of-function and/or gain-of-function mutations of key tricarboxylic acid (TCA) cycle enzymes could - at least in part - mechanistically account for dysfunctional mitochondrial oxidative metabolism in tumors as originally postulated by Warburg $[4,11,12]$. For example, lossof-function somatic mutations of succinate dehydrogenase (SDH) have been found in hereditary paragangliomas, pheochromocytomas, and renal cell cancers and those of fumarate hydratase (FH) have been detected in hereditary leiomyomatosis and renal cell cancer, inherited leiomyomas, and renal cell carcinoma $[4,11,12]$ : in this context, it is noteworthy that both SDH and FH are known to act as tumor suppressors [4,11]. Similarly, missense mutations in the genes encoding isocitrate dehydrogenase (IDH) thereby leading to changes in its catalytic specificity have been found in gliomas and acute myelogenous leukemia: the mutated IDH converts $\alpha$-ketoglutarate to its "oncometabolite" 2-hydroxyglutarate (2HG) $[4,11,12]$. Succinate and fumarate, the substrates of mutated SDH and FH respectively, and $2 \mathrm{HG}$, the "oncometabolite" of mutated IDH all inhibit $\alpha$-ketoglutarate-dependent dioxygenases that control the function of hypoxia-inducible factor 1 (HIF-1), a transcription factor that plays key roles in angiogenesis, metabolism, invasion and metastasis of cancer cells [4].
Studies that provided some evidence for:

(ii) Adaptation to hypoxia and shift to a glycolytic phenotype as being a viable mechanistic explanation for the Warburg hypothesis have revealed new facets of tumor cell metabolism extending beyond the original framework of the Warburg hypothesis $[3-8,10]$. These new facets include, but are not limited to, adaptive strategies of tumors and identification/characterization of metabolic phenotypes of cancer cells (see below).

\section{Adaptive Strategies and Metabolic Phenotypes of Cancer Cells}

\section{Adaptive strategies in intermediary metabolism of can- cer cells}

Different cancer types have adopted a set of strategies in their intermediary metabolism so as to enable them to survive, proliferate, and ultimately invade into their surrounding normal tissue. We will discuss their strategies in glucose oxidative metabolism, amino acid metabolism, and fatty acid synthesis.

\section{Adaptive glucose oxidative metabolism in tumors: The glycolytic shift}

As postulated in the Warburg hypothesis, the "glycolytic shift" in tumor metabolism is now known to be promoted by oncogenes (e.g., Ras and Myc) but inhibited by tumor suppressors (e.g., p53) as reflected in their underlying oncogenic transformation. Increased uptake of glucose into cancer cells (via upregulation of glucose transporters) facilitates and supports enhanced glycolysis in cancer cells $[3,4,7,10,11,13]$. This metabolic phenotype of cancer cells are the likely consequences of mutations and/or other alterations of oncogenes such as Ras and Myc and tumor suppressors such as p53. Furthermore, activation of Ras, Akt, and Myc and inactivation of p53 can contribute to the Warburg effect in a HIF-1-independent manner $[11,14]$.

Ras responds to activation by growth factors and transduces cell proliferation signals. In tumors, Ras is very frequently mutated results in its activation $[3,11]$. Ras mediates the activation of downstream pathways such as PI3K/Akt and MAPK [3]. Activation of growth factor/Ras mediated PI3K/Akt, enhances glycolysis via increased expression of the glucose transporter GLUT1, increased expression of genes involved in glycolysis, stimulation of hexokinase and phosphofructokinase activities, and increased association of hexokinases 1 and 2 (HK1 \& 2) with mitochondria [3,15]. MAPK also promotes glycolysis [3]. Similar to Ras and Akt, Myc is also activated by growth factors and it regulates many genes involved in cell proliferation $[3,11,14]$. Myc is known to be overexpressed in many tumor types and can stimulate aerobic glycolysis through increasing the expression of GLUT1 and lactate dehydrogenase A (LDHA) $[3,11,14]$.

The tumor suppressor p53 is a transcription factor that suppresses tumor development through regulating the expression of genes that induce transient cell cycle arrest, apoptosis, and senescence $[3,15-17]$. The TP53 gene encodes the p53 protein and is the 
most frequently mutated gene in human tumors [3,15-17]. Since p53 plays key roles in metabolic control through influencing the balance between glycolysis and oxidative phosphorylation [15-17], the glycolytic phenotype of cancer cells reflects a disruption of this metabolic balance in favor of reliance on glycolysis by cancer cells when they inactivate $\mathrm{p} 53$. The inactivation of p53 occurs mainly by two distinct mechanism

\section{a. by point mutation or}

b. via increased the degradation of p53 by deregulation of E3 ubiquitin ligase MDM2 [15-17]. Consequently, pharmacological activation of p53 function in cancer cells can and does lead to inhibition of their glycolytic enzymes, and ultimately contributes to their cell death [15].

As has already been alluded to above, one of the most successful adaptive strategies that tumors adopt in favor of the glycolytic shift is their ability to survive and prosper under hypoxic conditions that are often found in the core of rapidly growing solid tumors. Under hypoxic conditions, the prolyl hydroxylase domain protein 2 (PHD2) prolyl-hydroxylates proline residue at position 402 and/or 564 on HIF- $1 \alpha$ and prevents HIF- $1 \alpha$ from binding to the von Hippel-Landau tumor suppressor protein, and so prevents HIF- $1 \alpha$ from being degraded by the ubiquitin-mediated proteosomal mechanism [13]. The stabilized HIF1 alpha then binds to the constitutively expressed HIF-1 $\beta$, thereby forming the HIF-1 dimer [13]. Subsequently, the HIF-1 dimer is poised to activate the transcription of HIF1alpha regulated genes, including, GLUT 1 \& GLUT 3, HK1 and HK2 and other glycolytic enzymes, as well as pyruvate dehydrogenase kinase 1 (PDK1) [13,18]. PDK1 acts by inactivating the pyruvate dehydrogenase complex $[13,18]$. In addition to hypoxia, loss of tumor suppressors such as SDH, FH, ICDH (see above), activation of oncogenes such as Ras and Myc, growth factor signaling (e.g., by IGF, EGF, HER2 or PI3K), and reactive oxygen species (ROS) are all known to activate HIF-1 [3,4,11,13].

Complex functional interrelations exist between HIF-1 and mammalian target of rapamycin (mTOR) $[13,19]$. As mTOR is a serine-threonine protein kinase that phosphorylates ribosomal protein S6 kinase and eIF-4E binding protein, which enhances the translation rate of HIF-1 $\alpha$ [13], the translation rate of HIF- $1 \alpha$ in tumor cells is dependent on the activity of mTOR. Negative regulation of mTOR by activities occurs by upstream tumor suppressor proteins (e.g., PTEN, liver kinase B1 (LKB1), tuberous sclerosis complex 1 and 2 (TSC1, TSC2)) but positively regulated by upstream protein tyrosine kinases (e.g., epidermal growth factor receptor (EGFR)), and the oncogene Ras and its downstream signaling pathways, $[13,19,20]$. In the context of the regulation of rate of HIF- $1 \alpha$ translation in tumor cells, it is interesting to note that in many tumor types, the loss of function of tumor suppressors such as PTEN, LKB1, and/or TSC1/TSC2 due to their loss or mutations and the gain of function affecting protein tyrosine kinases, Ras and PI3K/ Akt signaling pathways contribute toward enhancing the mTOR-dependent HIF- $1 \alpha$ translation $[13,20]$. Consequently, this mTOR-induced, HIF-1-mediated mechanism favors the glycolytic switch in tumor cells via the upregulation of GLUT 1 \& GLUT 3, HK1 and HK2, and several other glycolytic enzymes as well as pyruvate dehydrogenase kinase 1 (PDK1), which then inactivates the pyruvate dehydrogenase complex, thereby limiting the flux of glycolytically-derived pyruvate into the TCA cycle $[13,18-20]$.

MTOR acts as an important regulatory gateway that controls whole body energy homeostasis through its differentiated functional roles in key metabolic organs/tissues such as liver, muscle, and adipose tissue, and consist of two structurally and functionally distinct complexes known as mTORC1 and mTORC2 [20,21]. The regulatory role of mTOR can be functionally delineated and distinctly assumed by mTORC1 and mTORC2, respectively. mTORC1 is the "nutrient sensor" and, together with its regulation of autophagy, exerts it signaling control on aging [21]. For example, mTORC1 is activated by growth factors, amino acids, and cell energy status to regulate protein synthesis, mitochondrial function, lipogenesis, ketogenesis, glucose homeostasis, and autophagy [21]. Commensurate with this important functional gating and its regulation, mTORC1 also integrates oncogenic growth signaling with the glycolytic switch, thereby aiding and abetting cell proliferation, growth, and survival in many cancer lineages $[20,21]$. On the other hand, when activated by growth factors, mTORC2 regulates glycolysis, gluconeogenesis, glycogenesis, and lipogenesis [21].

Evidence is accumulating that aberrant regulation of both cell growth and metabolism contributes significantly to cancer development and progression [1-4,7,10-15,20,21]. This conclusion is compatible with the observations that obesity and diabetes are risk factors for cancer and that diet can influence tumor growth [3,2022]. Also consistent with this conclusion are the clinical findings of decreased incidence of cancer in patients with type 2 diabetes mellitus (T2DM) when the patients were treated with metformin, which is considered as a first-line pharmacotherapy for T2DM $[23,24]$.

Metformin lowers blood glucose levels in people with diabetes and insulin resistance but does not exert this effect on healthy people [25]. Because there is good evidence implicating insulin resistance and hyperinsulinemia in the development and progression of cancer and many epidemiological studies strongly suggest T2DM individuals treated with metformin show a lower risk and improved outcomes with most common cancers [25,26], there is increasing interest and motivation to examine the putative anti-cancer effects of metformin in light of its mechanisms in regulation of glucose homeostasis $[24,25]$. As metformin is known to exert its effect by activating $5^{\prime}$ adenosine monophosphate (AMP)-activated protein kinase (AMPK) and AMPK is an important metabolic sensor in regulating energy metabolism in cells and tissues [24,25], metformin activates LKB1, a tumor suppressor, which phosphorylates and activates AMPK $[24,25]$. The metformin-activated AMPK then inhibits mTORC1 via the phosphorylation and stabilization of the tumor suppressor TCS2 [24,25]. Thus, the activation of AMPK by metformin upstream of mTOR requires the activation of LKB1. However, metformin can also inhibit mTOR by decreasing the levels of insulin or IGF1 independent of AMPK [25]. Consequently, AMPK-dependent and independent inhibition of mTOR induced by 
metformin can disrupt the mTOR-mediated integration of oncogenic growth signaling with the glycolytic switch, thereby inhibiting cancer cell survival, proliferation and cancer growth [20,21,24,25]. The inhibition of mTOR by metformin also decreases the rate of mTOR-dependent HIF- $1 \alpha$ translation $[13,19,21,24,25,27]$ : this negative regulation of HIF-1 by metformin likewise works against the glycolytic switch in tumor cells.

\section{Adaptive glucose oxidative metabolism in tumors: alter- ations in mitochondrial metabolism and mutations in \\ mitochondrial enzymes}

Despite the strong argument in favor of the successes of the glycolytic switch in cancer cells thereby allowing them to derive much, if not all, of the energy from aerobic glycolysis needed for their proliferation and growth [28-30], evidence has been accumulating that mitochondrial oxidative metabolism in tumor cells is by no means inactive or grossly dysfunctional as was originally hypothesized by Warburg and his coworkers. Two recent lines of investigations have provided increasing support for this conclusion:

I. Alterations in mitochondrial metabolism and mutations in mitochondrial enzymes in tumor cells and

II. Adaptive mitochondrial metabolism demonstrating the so-called "reversed Warburg effect."

As already discussed above in relation to the glycolytic switch in tumor cell metabolism, (i) alterations in mitochondrial metabolism and mutations in mitochondrial enzymes occur in a variety of tumor cells in parallel with or consequent to their glycolytic switch [4,8-12,15-20]. One example of alterations in mitochondrial oxidative metabolism can be found in tumor cells harboring the inactivated p53 through point mutation or increasing the degradation of the wild type (i.e., fully functional) [26-30] p53 by deregulation of E3 ubiquitin ligase MDM2 [15-17]. The loss-of-function of p53 is associated with their decreased oxygen consumptions and impaired mitochondrial respiration in the tumor cells; thereby favoring the glycolytic switch as noted above [15-17]. Several mechanisms could account for the decreased mitochondrial oxidative metabolism in tumor cells that contain loss-of-function p53. Two target genes of p53 important in regulation of energy metabolism are SCO2 (synthesis of cytochrome c oxidase 2) and TIGAR (TP53-induced glycolysis and apoptosis regulator). As SCO2 is a key regulator of cytochrome c oxidative complex essential for mitochondrial respiration, tumor cells with loss-of-function p53 can be expected to exhibit lowered mitochondrial respiration [16,31]. TIGAR functions to lower intracellular fructose-2, 6-bisphosphate resulting in decreased glycolysis and diverting glucose from glycolysis to the pentose phosphate pathway which produces more NADPH [16]. Furthermore, p53 also modulates glycolysis through the transcriptional regulation of other key glycolytic enzymes (e.g., phosphoglycerate mutase) [16]. Consequently, tumor cells with loss-of-function p53 can be expected to show enhanced glycolysis concomitant with decreased mitochondrial oxidative metabolism [16,31].

Consistent with the important role p53 plays in regulating energy metabolism is the recent finding that human glutaminase 2
(GLS2) is a novel, previously uncharacterized p53 target gene regulating energy metabolism and antioxidant function [32,33]. GLS2 is a mitochondrial glutaminase that converts glutamine to glutamate and allows its product glutamate to be further metabolized in mitochondria to produce ATP via oxidative phosphorylation (OXPHOS) $[32,33]$. Additionally, GLS2 regulates the production of glutathione (GSH) because its activity yields glutamate, which is a precursor of GSH; thus, GLS2 can increase reduced GSH but decrease ROS levels, thereby protecting cells from oxidative stress (e.g., $\mathrm{H}_{2} \mathrm{O}_{2}$-induced apoptosis) [32,33]. Furthermore, the observation that GLS2 levels are significantly decreased in hepatocellular carcinoma (HCC) and overexpression of this enzyme in tumor cells, including HCC cells, significantly lowers tumor cell colony formation strongly suggests a potential role for GLS2 in tumor cell growth suppression [32]. Other than in liver, GLS2 is also highly expressed in brain [32]. Thus, the recent finding [34] that GLS2 expression is lost in many brain tumors, including highly malignant glioblastomas and anaplastic astrocytomas, and restoration of the expression of this enzyme in glioblastoma cells inhibits tumor cell proliferation and migration is consistent with the notion that GLS2 plays an important role in tumor suppression, especially in tissues (e.g., liver and brain) that normally express this enzyme abundantly.

The hypoxic microenvironment in the core of a rapid growing solid tumor also favors their altered mitochondrial oxidative metabolism in parallel with enhanced glycolysis. As already discussed above, under hypoxic conditions, HIF- $1 \alpha$ becomes stabilized, thus allowing it to dimerize with the constitutively expressed HIF-1 $\beta$ and the HIF-1 dimer is then ready to activate the transcription of the genes that it regulates (e.g., GLUT1 \& GLUT3, HK1 and HK2, and PDK1) [13]. The upregulation of PDK1 leads to inactivation of the pyruvate dehydrogenase complex thereby resulting in decreased flux of glycolytically-derived carbon (in the form of pyruvate) into the TCA cycle and consequently also decreased flux through the TCA cycle $[13,18,19]$. Furthermore, in tumors with loss of tumor suppressors such as SDH, FH, and ICDH, activation of oncogenes such as Ras and Myc, together with activation of growth factor signaling (e.g., by IGF, EGF, HER2 or PI3K) and enhanced ROS production, their HIF-1 can be further activated beyond that expected of the hypoxic conditions alone leading to added enhancement of glycolysis but additional decreases in TCA cycle flux and mitochondrial oxidative metabolism $[3,4,11,13]$. Moreover, $p 53$ is a powerful negative regulator of HIF1- $\alpha$ because activation of p53 blocks the accumulation of HIF1- $\alpha$ (therefore its stabilization) in both hypoxia and normoxia and also inhibits HIF1 by inducing microRNA-107 (see [35] and references therein). Consequently, in tumors harboring loss-of-function p53, mitochondrial oxidative metabolism is expected to decrease concomitant with HIF1-induced enhancement of glycolysis.

Although mTORC1 is known to integrate oncogenic growth signaling with the glycolytic switch leading to proliferation, growth and survival in many cancer lineages $[20,21]$, how mTOR directly influences mitochondrial oxidative metabolism in tumor cells is unclear and awaits clarification. Nevertheless, we recently noted that the chronic hypoxia-induced alterations of key enzymes of glucose 
oxidative metabolism in developing mouse liver are mTOR dependent [19]. As already discussed above in relation to the glycolytic switch in tumor cell metabolism, (ii) adaptive mitochondrial metabolism demonstrating the so-called "reversed Warburg effect" constitutes a new development in the understanding of tumor cell metabolism [27]. Pavlides et al. [35] hypothesized that "epithelial cancer cells induce the Warburg effect (aerobic glycolysis) in neighboring stromal fibroblasts. These cancer-associated fibroblasts then undergo myo-fibroblastic differentiation and secrete lactate and pyruvate (energy metabolites resulting from aerobic glycolysis). Epithelial cancer cells could then take up these energy-rich metabolites and use them in the mitochondrial TCA cycle, thereby promoting efficient energy production (ATP generation via oxidative phosphorylation), resulting in a higher proliferative capacity. In this alternative model of tumorigenesis, the epithelial cancer cells instruct the normal stroma to transform into a wound-healing stroma, providing the necessary energy-rich micro-environment for facilitating tumor growth and angiogenesis. In essence, the fibroblastic tumor stroma would directly feed the epithelial cancer cells, in a type of host-parasite relationship." Pavlides et al. [35] have termed this newly discovered phenomenon the "Reverse Warburg Effect" $[35,36]$. Furthermore, they have systematically investigated cancer-associated fibroblasts and neighboring epithelial cancer cells in human breast cancers and found that the tumor cells and the stoma are metabolically coupled in a symbiotic or "host-parasite" relationship, thereby providing additional evidence in support of the "Reverse Warburg Effect" (see $[27,36]$ and references therein). Nevertheless, despite the fact that this novel concept is interesting and pathophysiologically relevant in tumor metabolism, whether or not this "Reverse Warburg Effect" occurs in all types of epithelial cancers- indeed in all tumor types -remains to be determined.

\section{Adaptive glucose metabolism via an alternative pathway}

The cancer cells require enhanced nucleotide generation to support their highly proliferative nature. The pentose phosphate pathway (PPP) that branches from the glycolysis converts glycolytic metabolites into pentose sugars that act as precursors for nucleotide synthesis [37]. In addition to nucleotide synthesis, the oxidative branch of the PPP serves as a major source of reduced nicotinamide adenine dinucleotide phosphate (NADPH) that regulates cellular redox balance through reduction of glutathione [38]. Notably, the activity of first enzyme of the oxidative PPP, glucose 6-phosphate dehydrogenase (G6PD), is upregulated in various tumors including bladder, prostate, breast, glioblastomas, cervical and other pre-neoplastic lesions [39-44]. The enzyme G6PD channels glycolytic metabolite glucose 6-phosphate into the oxidative PPP, and in the process generates NADPH from NADP. The upregulation of G6PD protects the cancer cells from oxidative damage induced cytotoxicity via production of NADPH [40]. The importance of G6PD can be gauged from the observations that overexpression of G6PD is sufficient to induce tumorigenesis in NIH3T3 cells, whereas downregulation of G6PD induces reactive oxygen species and growth retardation in fibroblast cells $[45,46]$. Overexpression of G6PD is also associated with enhanced tumor aggressiveness (as indicated by enhanced proliferation, promotion of invasion, increases in glucose uptake, cell cycle progression and reduce DNA damage) [47-50]. Regulation of cellular redox balance via G6PD overexpression also presents as an approach adopted by the cancer cells to overcome the cytotoxicity of chemotherapeutic drugs that rely on reactive oxygen species for their effectiveness [51-54].

Oncogenic signaling is also known to regulate the expression and activity of G6PD. Studies in multiple myeloma and hepatocellular carcinoma have demonstrated that increase in myc transactivation enhances G6PD transcription [55,56]. Through its regulation of G6PD, myc stimulates PPP flux, plays a crucial role in cellular redox balance, and hence promotes the tumor's resistance to chemotherapeutic agents [57]. Other oncogenic signaling molecules such as Akt and K-ras have also been shown to regulate the expression of G6PD $[40,58]$. Consequently, the control of PPP via oncogenic signaling molecules provides an attractive strategy to counter cancer growth and development. With reactive oxygen species playing a crucial role in cancer progression, deregulation of cellular redox balance via targeting the oxidative PPP has also been investigated. Pharmacological inhibition of G6PD by 6-aminonicotinamide (6AN) induces decreased NADPH production and leads to accumulation of ROS in cancer cells [40,59]. Other studies have also demonstrated the efficacy of 6-aminonicotinamide alone or in combination with DNA-damaging agents in lowering survival of cancer cells through alterations of cellular redox balance [60-63]; on the other hand, the ROS-independent mechanism mediating 6AN's efficacy have also been observed [64].

The non-oxidative pentose phosphate pathway derives its carbon from glycolytic fructose-6-phosphate and glyceraldehyde-3-phosphate and is important for generation of pentose sugars. The pentose sugars then act as precursors for nucleotide synthesis. Overexpression of key non-oxidative branch enzyme transketolase (TK) is associated with poor outcome in patients with ovarian and colorectal cancer $[65,66]$. In addition to the metabolic role, recent studies uncover the non-metabolic functions of TK wherein the enzyme can enter the nucleus and interact with cellular kinases and transcriptional factors that promote tumor aggressiveness $[67,68]$. Consequently, expression of TK is associated with enhanced cellular proliferation, invasion and metastasis $[69,70]$. Inhibition of TK leads to the promising outcome of inducing cell cycle arrest and apoptosis, reducing cancer cell migration, and enhancing the effects of chemotherapeutic agents and ionizing radiation [61,71-75].

Though promising, the current drug (i.e., 6AN) targeting the PPP exhibits high toxicity: thus, it cannot be approved for anti-tumor use in the clinic [76]. One explanation for the drug's high toxicity is that the PPP plays many important roles in controlling cellular redox and nucleotide biosynthesis. As mentioned earlier, the first enzyme of oxidative PPP is regulated by numerous oncogenic signaling molecules. A viable approach to target cancer cell PPP may lie in (i) identifying direct PPP inhibitors with low toxicity profiles and (ii) targeting signaling molecule (such as myc, Akt, etc) that regulate the PPP through gene transcription. Although new evidence continues to emerge highlighting the importance of PPP in tumor progression, a better understanding of the oncogenic/met- 
abolic switch that decides the fate of glucose either to enter glycolysis or the pentose phosphate pathway would pave the way for the personalized approach to treat cancers.

\section{Adaptive amino acid metabolism in tumors}

The recent advances in the understanding of adaptive tumor metabolism have not only revealed novel molecular and signaling mechanisms in different tumors to affect their glycolytic switch but also uncovered new facets of altered tumor metabolism to provide carbon and nitrogen sources to satisfy the biosynthetic needs of rapidly proliferating tumor cells $[3,4,10,16,21,28,31,77-80]$. A case in point is the adaptive amino acid metabolism in tumors where their glycolytic switch together with the not inactive mitochondrial oxidative metabolism channel glucose-derived carbon into synthesis of amino acids [3,4,10,16,21,28,31,77-80]. For example, in addition to forming alanine from the glycolytically-derived pyruvate, tumors can synthesize serine and glycine from glycolytic intermediates [3]. Furthermore, the recent finding of the existence of an alternative glycolytic pathway that "decouples ATP production from phosphoenolpyruvate-mediated phosphor transfer, allowing for the high rate of glycolysis to support anabolic metabolism observed in many proliferating cells" [79] can attest to the versatility of tumors' synthetic capabilities. Nutritional type of studies that purported to demonstrate tumor cells exhibit an "addiction to glutamine" for its energetic and biosynthetic needs have uncovered several novel roles that glutamine may play in tumor metabolism in sustaining tumors' survival, proliferation, and perhaps even migration and metastasis in the face of warding off autophagy and apoptosis $[32-34,77,79]$. Because of its high circulating level, which can reach $1 \mathrm{mM}$, under physiological conditions, glutamine can serve as the key amino acid that mediates the inter-organ transfer of both carbon and nitrogen $[10,77]$. Thus, circulating glutamine can be exploited by rapidly growing tumors to sustain their growth. More importantly, adaptive glutamine metabolism in tumors has conferred on them unusual capacity to survive and proliferate. As already discussed above, because glutaminase 2 (GLS2) may play an important role in tumor suppression [32,34], that GLS2 expression is lost in many brain tumors, including highly malignant glioblastomas and anaplastic astrocytomas [34] and GLS2 levels are significantly decreased in hepatocellular carcinoma [32] allows these types of tumors more freedom to proliferate unchecked.

Tumor cells can survive periods of nutrient depletion and/or exposure to targeted therapies through alterations in the route they metabolize glutamine. For example, when glucose is abundant, tumor cells employ transamination reactions in producing $\alpha$-ketoglutarate from glutamine [77]. On the hand, as glucose becomes scant, tumors employ glutamate dehydrogenase (GDH) to produce $\alpha$-ketoglutarate from glutamine [77]. The choice between the two metabolic routes is controlled indirectly by oncogenic signaling pathways as such pathways tend to drive glucose metabolism in tumors: for example, inhibitors of PI3K/Akt/mTOR signaling pathway render cells dependent on GDH for survival [77].

Their metabolism of glutamine yields glutamate, a key precursor for the synthesis of the antioxidant glutathione (GSH; see above)
$[32-34,77]$ that protects cells from oxidative and oncogenic stress. Additionally, the flux of glutamine-derived carbon through the TCA cycle and then through the malic enzyme will reduce NADP+ to NA$\mathrm{DPH}$, which is required to regenerate reduced GSH [77]. Glutamine is also important in regulating tumor cell autophagy. Two such mechanisms have been characterized. (i) When mTORC1 is active, glutamine tends to suppress autophagy activity by facilitating the transport of mTORC1-activating amino acids such as leucine, [77]. (ii) Ammonia, a product formed from glutamine by glutaminase, can stimulate autophagy in tumor cells at lower levels $(\leq 2 \mathrm{mM})$ but suppress autophagy in tumor cells at higher levels ( $\geq 10 \mathrm{mM})$ [77]. Another metabolite produced by tumor cells from glutamine metabolism is pyruvate, which is then eventually converted to lactate [77]: consequently, in addition to the glycolytic shift, tumors can also produce lactate through glutamine metabolism. The increased production of lactate by tumors generates an acidic microenvironment that promotes tumor cell invasion and metastasis [77].

\section{Adaptive fatty acid metabolism in tumors}

Compared with adaptive amino acid metabolism in tumors that of fatty acid metabolism is much less well characterized. With the exception of the oncogenic role of fatty acid synthase (FASN), the adaptive fatty acid metabolism in tumors can only be described in very sketchy terms as the underlying molecular and signaling mechanisms are still poorly understood. Nevertheless, along with the Warburg effect, increased de novo synthesis of lipids is ubiquitous in various tumors while most normal cell types (with the exception of liver and adipose tissue) exhibit very low rates of lipid synthesis [20]. The sterol regulatory element-binding protein (SREBP) transcription factors (SREBP1a, SREBP1c, and SREBP2) are important regulators of genes involved in lipid and sterol synthesis [20]. Although SREBPs are normally tightly regulated, the mechanisms of their regulation have not been fully elucidated [20]. Nevertheless, the PI3K-Akt pathway is known to upregulate lipogenic gene expression in a SREBP-dependent fashion and mTORC1 has been noted to be the downstream effector responsible for SREBP activation [20]. Thus, in tumors where the Ras/PI3K/Akt/mTOR pathway is activated, their adaptive fatty acid metabolism may be sensitive to specific inhibitors that target this pathway $[20,22]$. For example, as mTOR plays a critical role in cell growth and tumorigenesis in a variety of tumors, metformin (see earlier discussion on metformin action) can exert its anti-tumor effects via inhibiting mTOR by decreasing the levels of insulin or IGF1 independent of AMPK [25], thereby also decreasing the tumors' rates of de novo lipid synthesis [80].

Among the adaptive molecular mechanisms of fatty acid metabolism in tumors, the oncogenic role of fatty acid synthase (FASN) is the most studied. Nevertheless, this role of FASN is far from being comprehensively understood [81]. FASN is an enzyme that controls lipid biosynthesis by producing long-chain fatty acids from acetyl-CoA and malonyl-CoA. During glucose oxidative metabolism in tumors, acetyl-CoA is produced, and tumors use it as precursor for their lipid biogenesis [38,80,81]. In fast-growing tumors, fatty acids are synthesized de novo to supply their functional needs (i.e., lipids 
for membrane biogenesis, ATP production via $\beta$-oxidation of fatty acids, and lipid modifications of proteins) $[38,41]$. Consistent with the functional needs for lipids in tumors are the findings that FASN is highly expressed in numerous cancers, including bladder, breast, colorectal, endometrial, esophageal, hepatocellular, lung, oral, prostate, thyroid, tongue, and pancreatic and gastric carcinomas as well as other tumors [81]. Additionally, enhanced FASN expression has also been found in some benign and pre-invasive lesions of breast, colon, lung, prostate and stomach [41]. Because FASN overexpression is associated with poor prognosis and decreased patient survival in many cancer types and FASN plays an important role in tumor cell development and survival, that FASN may act as a metabolic oncogene in cancer cells is beginning to be recognized but the underlying molecular mechanisms remain to be completely delineated [81,82]. Several lines of evidence are in support of the role of FASN as a metabolic oncogene.

A. Steroid hormones (e.g., estrogens), growth factors (e.g., EGFR and ERBB2) and the PI3K-Akt signaling pathways are known to modulate FASN expression in several types of cancer [81,82].

B. Pharmacological inhibition of FASN activity using cerulenin, a characterized inhibitor of FASN, induces a selective cytotoxicity in cancer cells by lowering their fatty acid synthesis: this inhibition of fatty acid synthesis leads to delayed progression of human breast, ovarian, and prostate xenografts and suppression of liver metastasis in colon cancer xenograft model (see [82] and references therein).

C. There is new evidence that $G$ protein-coupled estrogen receptor (GPER) should be included among the transduction mediators involved by estrogens in controlling FASN expression and activity in tumors and tumor-associated fibroblasts that strongly contribute to tumor progression [82]. Consequently, the above findings in support of the role of FASN as a metabolic oncogene suggest that FASN should be considered as a suitable target for anti-cancer drug discovery.

\section{Adaptive strategies and metabolic phenotypes of tu- mors: Some conclusions and a synthesis}

As evident from the above discussion of the various strategies that different types of tumors appear to have adopted to sustain their survival, proliferation and ultimately metastasis, several tentative conclusions can be arrived at and some holistic synthesis can be attained as to how this state-of-the-art understanding of the diversity of tumor metabolism can inspire new strategies for anti-cancer drug discovery.

Three tentative conclusions are as follows.

A. In addition to the adaptive metabolic strategies and phenotypes in tumors considered above, others need also be considered. For example, some researchers have argued for the advantages of targeting mitochondrial oxidative and redox homeostasis not emphasized in this review as well as other TCA cycle enzymes such as IDH, FH, and SDH that have been only very briefly discussed herein or not discussed at all [11,12,14,83-85]. However, while such targets are plausible $[11,12,14,83-85]$, they have not been report- edly investigated to nearly the same detail as those discussed in this review.

B. As we $[10]$ and others $[3,4,12-14,83,85]$ have maintained, cancer is not one disease but a multitude of syndromes. In addition to the fact cancer is not a single disease, there is increasing evidence in support of the following hypothesis. "Independently of whether malignant tumors are initiated by a fundamental reprogramming of gene expression or seeded by stem cells, "waves" of gene expression that promote metabolic changes that occur during carcinogenesis, beginning with oncogene-mediated changes, followed by hypoxia-induced factor (HIF)-mediated gene expression, both resulting in the highly glycolytic "Warburg" phenotype and suppression of mitochondrial biogenesis. Because high proliferation rates in malignancies cause aglycemia and nutrient shortage, the third (second oncogene) "wave" of adaptation stimulates glutaminolysis, which in certain cases partially re-establishes oxidative phosphorylation; this involves the LKB1-AMPK-p53, PI3K-Akt-mTOR axes and MYC dysregulation. Oxidative glutaminolysis serves as an alternative pathway compensating for cellular ATP. Together with anoxic glutaminolysis it provides pyruvate, lactate, and the NADPH pool (alternatively to pentose phosphate pathway). Retrograde signaling from revitalized mitochondria might constitute the fourth "wave" of gene reprogramming. In turn, upon reversal of the two Krebs cycle enzymes, glutaminolysis may partially (transiently) function even during anoxia, thereby further promoting malignancy. The history of the carcinogenic process within each malignant tumor determines the final metabolic phenotype of the selected surviving cells, resulting in distinct cancer bioenergetic phenotypes ranging from the highly glycolytic "classic Warburg" to partial or enhanced oxidative phosphorylation" [86]. According this hypothesis [86], cells within a tumor are likely to exhibit diverse, or at least different, metabolic phenotypes. Thus, it is not realistic and practicable just to strategize about anti-cancer drug discovery around a single target: after all, "one size cannot fit all"!

C. Despite the wealth of recent studies that purport to characterize the metabolic phenotypes of diverse tumor types, there are as yet many uncertainties regarding how one can delineate metabolic phenotypes of tumor cells from the normal phenotypes of their corresponding non-cancerous counterparts $[3,10,21,35,36,7$ $7,78,80,83,85,86]$. For example, metabolic adaptation and reprograming occurs not only in transformed cells but also in non-transformed cells and/or tissues, thereby raising the question [3]: "Is there a generally recognizable metabolic profile of tumor cells that differ from that of non-cancerous but proliferating cells?"

From the considerations and discussion of issues regarding adaptive tumor metabolism and metabolic phenotypes of cancer cells, two points of consensus have emerged.

(i) More detailed and systematic investigation of metabolism of any given and/or chosen type of tumor and the corresponding metabolic phenotype of its normal cell/tissue counterpart need to be conducted to more definitively distinguish the readily recognizable metabolic profile of a tumor from that of its non-cancerous but proliferating normal counterpart. 
(ii) One interim translational solution that has emerged is that targeting tumor metabolism in anti-cancer drug discovery could advantageously adopt the multi-target approach.

\section{Uncovering Putative Anti-Cancer Drug Targets}

Given that the diversity of tumors exhibits multiple adaptive metabolic gene expression signatures and phenotypes, as has already been detailed above, targeting tumor metabolism for anti-cancer drug discovery ought to address this multitude of altered metabolism in tumors to ascertain that the outcome of the enterprise will be drug(s) that will be efficacious but with minimal or no toxicity. We have already highlighted above the utility and advantages of adopting the multi-target approach (e.g., target a pathway rather than a single target such as an enzyme or a transporter). Employing this approach, we will briefly discuss selected "drug-ofchoice" examples to illustrate the proof-of-concept considerations as some of our examples are among drugs that are either in pre- or different phases of clinical trials [14]. Our "drug-of-choice" examples include 3-bromopyruvate [7,10,87], metformin [23-27,36], inhibitors of both PI3K and mTOR [22,88,89], and inhibitors of FASN [81]. Our rationale for choosing these as examples of our "drugof-choice" is that they appear to exert their anti-tumor effects on multiple targets and as such they have been shown to have added advantages when used in combination therapies for treating different types of cancer.

\section{3-bromopyruvate (3-BP)}

Irrespective whether or not the Warburg hypothesis is still tenable, given the renaissance of research on tumor metabolism it has inspired in the last decade or so, evidently actively growing tumors do depend on glycolysis to a greater or lesser extent for energy and carbon for biosynthetic purposes for their survival, proliferation and growth [3-8,10-14]. It is, therefore, not surprising that tumor glycolysis is one of the earliest developed target(s) for anti-cancer drug discovery and hexokinase (HK), the enzyme that catalyzes the first and rate-limiting step in glycolysis, has become one of the key targets, if not the prime target [3-8,10-14]. Inhibitors of HK, such as 2-deoxyglucose (2-DG), lonidamine (a derivative of indazole-3-carboxylic acid) and 3-bromopyruvate (3-BP), have been employed in pre-clinical and/or early clinical trials for treating several tumor types [14]. Both 2-DG and lonidamine have been employed in phase I/II clinical trials, especially in combination with other more established chemotherapeutic agents, for treating solid tumors (including breast, ovarian and lung cancer) and glioblastoma multiforme [14]. Because of its anti-cancer potential in pre-clinical studies and its reportedly low toxicity [87,90], 3-BP is poised in being launched into human clinical trials for treating tumors [91-93]. Consequently, 3-BP is one of the desired candidates for human clinical trials in intra-arterial therapy for hepatocellular carcinoma [51-53]. Our choice for 3-BP for consideration as a "drug-of-choice" example hinges on the fact 3-BP exerts its anti-cancer effects on multiple targets other than its well-characterized effect on $\mathrm{HK}$ alone $[7,10,14,87,90]$.

As a recognized inhibitor of glycolysis, in addition to inhibiting HK and disrupting HK2's binding to mitochondria thereby prevent- ing HK2 from blocking apoptosis control, 3-BP is also known to inhibit several glycolytic enzymes downstream from HK [87]. Among several glycolytic enzymes in rat liver cytosol investigated, 3-BP more selectively inhibits glyceraldehyde-3-phophate dehydrogenase (GAPDH), leading to the blockade of both of the ATP-producing steps downstream of GAPDH [87]. Moreover, GAPDH has been demonstrated to be a multi-functional protein that can affect proliferation of cancer cells: in human hepatocellular carcinoma, increased GAPDH expression is associated with enhanced glycolytic capacity facilitating tumor progression as there is a link between GAPDH upregulation and the promotion of survival mechanisms in cancer cells and their resistance to chemotherapeutic agents $[94,95]$. Induction of cellular stress also contributes to 3-BP-induced apoptosis [95]. Because 3-BP is selectively taken up by tumor cells via the monocarboxylate transporters (MCTs) and MCTs are frequently overexpressed in cancer cells, this preferential uptake of 3-BP allows the differential targeting of 3-BP to cancer rather than to normal cells [95].

In isolated mitochondria, mM levels of 3-BP inhibit succinate-supported oxygen uptake [87]. Thus, 3-BP-medicated inhibition of succinate dehydrogenase ( $\mathrm{SDH}$ ) may lead to induction of ROS (especially superoxide) and tumor cell death: along the way, the SDH inhibition is also expected to impair glutaminolysis thereby compromising the tumors' glutamine-dependent functions [47]; also see the discussion of tumor dependence on glutaminolysis above.

\section{Metformin}

Metformin has two distinct advantages as a selected "drug-ofchoice" $[23-27,36]$ in preclinical studies: these advantages prompted us to exploit it further for anticancer drug discovery. They are as follows.

1) As metformin has been employed to lower blood glucose levels in individuals with diabetes and is a first-line treatment for type 2 diabetes mellitus (T2DM), humans are generally known to tolerate this drug quite well and adverse effects due to its intake are uncommon or minimal if present [25]. Consequently, metformin is a safe drug for humans [25].

2) There has been increasing epidemiological and other evidence indicating that diabetic patients who have been treated with metformin show a lower incidence of and mortality from cancer and treatment of T2DM patients with metformin is associated with a lowered mortality due to several types of cancer including, but not limited to, colorectal, hepatocellular and pancreatic carcinomas [25].

Metformin is largely known to exert its major effects through activation of AMPK, a metabolic sensor responsible for controlling energy metabolism [24-27]. Metformin activates the tumor suppressor LKB1, which then phosphorylates and activates AMPK $[24,25]$. Upon its activation, AMPK inhibits mTORC1; however, metformin can also inhibit mTOR independent of AMPK by decreasing insulin or IGF1 levels [25]. The inhibition of mTOR induced by metformin leads to disruption of the integration of mTOR-mediated oncogenic signaling and glycolytic switch resulting in inhibition of 
tumor survival and growth $[20,21,24,25]$. This inhibitory effect of metformin on mTOR also decreases the mTOR-dependent HIF-1 $\alpha$ translation: the latter mechanism also works against the glycolytic switch in tumors $[13,19-21,24,25]$. It is noteworthy that chronic activation of AMPK by metformin alters mitosis and also activation of p53 and cellular senescence: thus, the metformin-induced inhibition of oxidative phosphorylation is suggested to increase glycolysis and autophagy in cells harboring wild-type (i.e., normal) p53 while metformin-treated cancer cells harboring mutated p53 are unable to reprogram their metabolism and such cancer cells undergo apoptosis [25]. Additionally, metformin is also known to be a mitochondrial complex I inhibitor and prevents OXPHOS in breast cancer cells [27]. Even though the molecular mechanisms underlying the anti-cancer effects of metformin are far from being completely understood, there is increasing evidence supporting the notion that the classic anti-diabetic drug exerts its effects on multiple cell survival, proliferation and invasion signaling pathways in tumors [13,19-21,24,25,27,36], thereby indicating metformin's multi-target potential as an anti-tumor agent. Consistent with this conclusion is the fact metformin is currently under phase I and II clinical trials for its anti-tumor potential for treating solid tumors and lymphoma [14].

\section{Inhibitors of both PI3K and mTOR}

PI3K and mTOR are two key components of the PI3K/Akt/ mTOR signaling pathway that regulates a diverse range of cell physiology and mechanism including, but not limited to, proliferation, motility, metabolism, differentiation, apoptosis, autophagy and exocytosis [22, 88, and 89]. Because this signaling pathway is constitutively active in many tumor types in which it negatively affects responses to various therapies, developing inhibitors that target both PI3K and mTOR in the same pathway has the added advantage over developing just the single-target inhibitors such as PI3K inhibitors or mTOR inhibitors (e.g., rapamycin) $[22,88,89]$. The first released ATP-competitive kinase inhibitor of mTOR that also inhibits the enzymatic activity of PI3K p110 isoforms is PI-103. PI-103 is a morpholino quinazoline derivative and a pan-class $1 \mathrm{PI} 3 \mathrm{~K}$ inhibitor with IC50 values in the low nM range: it exhibits good selectivity over the rest of the human kinome [96]. Several other similar compounds have been subsequently released (e.g., NVP-BEZ235, XL765, PKI587, PF-04691502, WJD0008, PKI-402, and GNE-477) [89]. These dual PI3K/mTOR inhibitors exhibit significant, concentration-related inhibition of cell proliferation and induction of apoptosis in a wide variety of cancer cell lines, including those containing PI3K p110 $\alpha$ activating mutations [49]. The in vitro activities of these dual $\mathrm{PI} 3 \mathrm{~K} / \mathrm{mTOR}$ inhibitors translate well in in vivo models of human cancer xenografted in mice [89]. The compounds were well tolerated and induced tumor stasis or even regression when given orally [89]. As the pre-clinical studies of such compounds have yielded quite favorable outcome, a few of them (e.g., NVP-BEZ235) are currently employed in phase I/II clinical trials in cancer patients [89].

\section{Inhibitors of fatty acid synthase (FASN)}

Because increased de novo synthesis of lipids is ubiquitous in a variety of tumors while most normal cell types exhibit very low rates of lipid synthesis [20] and FASN is an enzyme that controls lipid biosynthesis as well as a putative metabolic oncogene [41,42; also see section on Adaptive fatty acid metabolism in tumors above], inhibitors of FASN become our "drugs-of-choice." Thus, FASN inhibitors can be expected to exert their anti-tumor effects through acting on multiple targets [81]. Small-molecule FASN inhibitors such as cerulenin, C75, and orlistat (a US FDA-approved pancreatic lipase inhibitor, originally developed as an anti-obesity drug) have been shown to induce apoptosis in multiple cancer cell lines and to delay tumor growth in some tumor xenograft models although the underlying mechanisms are incompletely understood [81]. The small-molecule FASN inhibitors exhibit pharmacological limitations and weight loss as an adverse/side effect thereby precluding them from further development as systematic drugs [81]. Several new compounds have been developed recently by pharmaceutical companies and they show good potential as FASN inhibitors but their efficacy as anti-tumor agents remains to be determined [81]. Nevertheless, novel sources of FASN inhibitors such as green tea and dietary soy may be further exploited for their anti-tumor and cancer prevention potentials [81].

\section{Conclusion}

The renewed interests in the Warburg hypothesis or Warburg effect have motivated investigators to identify mechanistic explanations for the two key issues of the hypothesis - (i) dysfunctional mitochondrial oxidative metabolism and (ii) adaptation to hypoxia and shift to a glycolytic phenotype (i.e., the "glycolytic switch"). Consequently, these recent advances allow the identification of adaptive metabolic phenotypes among the diverse varieties of tumor cell types studied. Moreover, these advances have led to the discovery of the new phenomenon in tumor cell metabolism ingeniously called "Reverse Warburg Effect." These adaptive metabolic phenotypes in tumors include, but are not limited to: Adaptive glucose oxidative metabolism: the glycolytic switch; adaptive glucose oxidative metabolism: Alterations in mitochondrial metabolism and mutations in mitochondrial enzymes; adaptive glucose metabolism via an alternative pathway; adaptive amino acid metabolism; and adaptive fatty acid metabolism.

In discussing the adaptive metabolic phenotypes in tumor cells and, in parallel, the underlying signaling and other molecular mechanisms, three conclusions have been arrived at. (i) Other adaptive metabolic strategies and phenotypes in tumors need to be considered even though they are not as well documented as those discussed in this review. (ii) Within a tumor, the cells therein may exhibit "waves" of gene expression that promote metabolic changes that occur during carcinogenesis, thereby embodying the notion that within a tumor, cells are likely to exhibit different and/ or diverse metabolic phenotypes, rendering it unrealistic to strategize about anti-cancer discovery around a single drug target. (iii) Despite the impressive advances in tumor metabolism research to date, the following question still cannot be answered: "Is there a generally recognizable metabolic profile of tumor cells that differ from that of non-cancerous but proliferating cells?"

We have highlighted the utility and advantages of adopting the multi-target approach and briefly discuss selected "drug-of- 
choice" examples to illustrate the proof-of-concept considerations as some of our examples are among drugs that are either in preor different phases of clinical trials. Our "drug-of-choice" examples include 3-bromopyruvate, metformin, inhibitors of both PI3K and mTOR, and inhibitors of FASN. Our rationale for choosing these as examples of our "drug-of-choice" is that they appear to exert their anti-tumor effects on multiple targets and as such they have been shown to have added advantages when used in combination therapies for treating different types of cancer. We have emphasized several novel but practicable trends of research that will facilitate targeting tumor metabolism for anti-cancer drug discovery to follow the dictum "from bench to the bedside." We hope our forward-looking framework for discussing adaptive metabolic phenotypes in tumors will further generate positive as well as productive discussion and open new avenues for rendering targeting tumor metabolism for anti-cancer drug discovery a realizable reality.

\section{Future Perspective}

In the last decade, major advances in understanding tumor metabolism have been made as a direct result of the renaissance of research interests in the Warburg Hypothesis or Warburg Effect. From the critical evaluation and discussion of the current issues pertaining to adaptive tumor cell metabolism and phenotypes and targeting tumor metabolism for anti-cancer drug discovery, three distinct trends of research are beginning to emerge. These trends appear to be forward-looking and have the potential of being ground-breaking and undoubtedly productive. The first area continues to be off shoots from further research to investigate the Warburg Hypothesis or Warburg Effect, especially in two directions. (i) The hypothesis put forward by Smolková and collaborators [46] regarding "Waves of gene regulation suppress and then restore oxidative phosphorylation in cancer cells" highlights the importance of the realization that the cells within a solid tumor exhibit a variety of adaptive metabolic phenotypes. Consequently, further research and future advances will undoubtedly provide new insights into the diversity of metabolic phenotypes with a solid tumor $[43,46]$. (ii) The newly discovered metabolic phenomenon where "the fibroblastic tumor stoma would directly feed the epithelial cancer cells, in a type of host-parasite relationship" led to the mechanistically important proposal of the "Reverse Warburg Effect" [35,36]. Ongoing and future studies will clarify to what generalized degree this metabolic interrelationship exist between stromal fibroblasts and neighboring epithelial cancer cells and if this effect occurs consistently and regularly in all types of epithelial cancers, if not in all types of different tumors.

The second area pertains to the as yet unresolved but pathophysiologically important question $[3,10,21,35-38,40,43,45,46]$ : "Is there a generally recognizable metabolic profile of tumor cells that differ from that of non-cancerous but proliferating cells?" Indeed, current as well as future advances will definitely provide an answer (or answers) to this germane question. The third area relates to the emergence of one interim translational solution in targeting tumor metabolism for anti-cancer drug discovery. This area has highly promising translation potential. This research and translational activities can and should advantageously adopt the multi-target approach, especially in combination therapies where new multi-target anti-cancer agents under trial are employed together with already approved chemotherapeutic drugs to fight cancers $[14,22,23,25,41,47,49,55,56]$.

\section{Acknowledgement}

James CK Lai was supported, in part, by a DoD USAMRMC Project Grant (Contract \#W81XWH-07-2-0078) and small project grant from MSTMRI.

\section{Financial \& Competing Interests Disclosure}

The authors have no other affiliations or financial involvement with any organization or entity with a financial interest in or financial conflict with the subject matter or materials discussed in the manuscript apart from those disclosed.

\section{References}

1. Warburg O, Wind F, Negelein E (1927) The metabolism of tumors in the body. J Gen Physiol 8(6): 519-530.

2. Warburg O (1956) On the origin of cancer cells. Science 123(3191): 309314.

3. Muñoz-Pinedo C, El Mjiyad N, Ricci JE (2012) Cancer metabolism: Current perspectives and future directions. Cell Death and Disease 3: e248.

4. Semenza GL (2011) A return to cancer metabolism. J Mol Med (Berl) 89(3): 203-204.

5. Stegh AH, Chin L, Louis DN, DePinho RA (2008) What drives intense apoptosis resistance and propensity for necrosis in glioblastoma? A role for Bcl2L12 as a multifunctional cell death regulator. Cell Cycle 7(18): 2833-2839.

6. Lino M, Merlo A (2009) Translating biology into clinic: The case of glioblastoma. Curr Opin Cell Biol 21(2): 311-316.

7. Bhardwaj V, Rizvi N, Lai MB, Lai JCK, Bhushan A (2010) Glycolytic enzyme inhibitors affect pancreatic cancer survival by modulating its signaling and energetics. Anticancer Res 30(3): 743-749.

8. Ordys BB, Launay S, Deighton RF, McCulloch J, Whittle IR (2010) The role of mitochondria in glioma pathophysiology. Mol Neurobiol 42(1): 64-75.

9. Czarnecka A, Bartnik E (2009) Mitochondrial DNA mutations in tumors. In: Apte SP, Sarangarajan R (Eds.), Cellular respiration and carcinogenesis. Humana Press, New York, USA, pp.119-130.

10. Jain A, Lai JCK, Chowdhury GMI, Behar K, Bhushan A (2011) Glioblastoma: Current chemotherapeutic status and need for new targets and approaches. In: Abujamra AL (Ed.), Brain tumors: Current and emerging therapeutic strategies. InTech, Rijeka, Croatia, pp. 145-176.

11. Dang CV, Hamaker M, Sun P, Le A, Gao P (2011) Therapeutic targeting of cancer cell metabolism. J Mol Med 89(3): 205-212.

12. Frezza C, Pollard PJ, Gottlieb E (2011) Inborn and acquired metabolic defects in cancer. J Mol Med 89(3): 213-220.

13. Semenza GL (2010) HIF-1: Upstream and downstream of cancer metabolism. Curr Opin Genet Dev 20(1): 51-56.

14. Zhao Y, Liu H, Riker AI, Fodstad O, Ledoux SP, et al. (2011) Emerging metabolic targets in cancer therapy. Front Biosci 16: 1844-1860.

15. Zawakca PJ, Grinkevich VV, Hünten S, Nikulenkov F, Gluch A, et al. (2011) Inhibition of glycolytic enzymes mediated by pharmacologically active p53: Targeting Warburg effect to fight cancer. J Biol Chem 286(48): 41600-41615. 
16. Feng Z, Levine AJ (2010) The regulation of energy metabolism and the IGF-1/mTOR pathways by the p53 protein. Trends Cell Biol 20(7): 427434.

17. Reinhardt HC, Schumacher B (2012) The p53 network: Cellular and systemic DNA damage responses in aging and cancer. Trends Genet 28(3): 128-136.

18. Zhou, D, Xue J, Lai JCK, Schork NJ, White KP, et al. (2008) Mechanisms underlying hypoxia tolerance in drosophila melanogaster: Hairy as a metabolic switch. PLoS Genetics 4(10): e1000221.

19. Dukhande VV, Sharma GC, Lai JCK, Farahani R (2011) Chronic hypoxiainduced alterations of key enzymes of glucose oxidative metabolism in developing mouse liver are mTOR dependent. Mol Cell Biochem 357(12): $189-197$.

20. Yecies JL, Manning BD (2011) mTOR links oncogenic signaling to tumor cell metabolism. J Mol Med 89(3): 221-228.

21. Cornu M, Albert V, Hall MN (2013) mTOR in aging, metabolism, and cancer. Curr Opin Genet Dev 23(1): 53-62.

22. McCubrey JA, Steelman LS, Chappell WH, Abrams SL, Montalto G, et al. (2012) Mutations and deregulations of Ras/Raf/MEK/ERK and PI3K/ PTEN/Akt/mTOR cascades which alter therapy response. Oncotarget 3(9): 954-987.

23. Cheong JH, Park ES, Liang J, Dennison JB, Tsavachidou D, et al. (2011) Dual inhibition of tumor energy pathways by 2-deoxyglucose and metformin is effective against a broad spectrum of preclinical cancer models. Mol Cancer Ther 10(12): 2350-2362.

24. Micic D, Cvijovic G, Trajkovic V, Duntas LH, Povovina S (2011) Metformin: Its emerging role in oncology. Hormones (Athens) 10 (1): 5-15.

25. Del Barco S, Vazquez-Martin A, Cufi S, Oliveras-Ferraros C, BoschBarrera J, et al. (2011) Metformin: Multi-faceted protection against cancer. Oncotarget 2(12): 896-917.

26. Godsland IF (2010) Insulin resistance and hyperinsulinemia in the development and progression of cancer. Clin Sci (Lond) 118: 315-332.

27. Sanchez-Alvarez R, Martinez-Outschoom UE, Lamb R, Hulit J, Howell A, et al. (2013) Mitochondrial dysfunction in breast cancer cells prevents tumor growth. Cell Cycle 12(1): 172-182.

28. Vander Heiden MG, Cantley LC, Thompson CB (2009) Understanding the warburg effect: The metabolic requirements of cell proliferation. Science 324(5930): 1029-1033.

29. Kaelin Jr WG, Thompson CB (2010) Q\&A: Cancer: Clues from cell metabolism. Nature 465(7298): 562-564.

30. Levine AJ, Puzio-Kuter AM (2010) The control of the metabolic switch in cancers by oncogenes and tumor suppressor genes. Science 330(6009): 1340-1344

31. Fogg VC, Lanning NJ, MacKeigan JP (2011) Mitochondria in cancer: At the crossroads of life and death. Clin J Cancer 30(8): 526-539.

32. Hu W, Zhang C, Wu R, Sun Y, Levine A, et al. (2010) Glutaminase 2, a novel p53 target gene regulating energy metabolism and antioxidant function. Proc Natl Acad Sci USA 107(16): 7455-7460.

33. Suzuki S, Tanaka T, Poyurovsky MV, Nagano H, Mayama T, et al. (2010) Phosphate-activated glutaminase (GLS2), a p53-inducible regulator of glutamine metabolism and reactive oxygen species. Proc Natl Acad Sci USA 107(16): 7461-7466.

34. Szeliga M, Obara-Michlewska M, Matyja E, Łazarczyk M, Lobo C, et al. (2009) Transfection with liver-type glutaminase cDNA alters gene expression and reduces survival, migration and proliferation of T98G glioma cells. Glia 57(9): 1014-1023.

35. Pavlides S, Whitaker-Menezes D, Castello-Cros R, Flomenberg N, Witkiewicz AK, et al. (2009) The reverse warburg effect: aerobic glycolysis in cancer associated fibroblasts and the tumor stroma. Cell Cycle 8(23): 3984-4001.
36. Whitaker-Menezes D, Martinez-Outschoorn UE, Flomenberg N, Birbe RC, Witkiewicz AK, et al. (2011) Hyperactivation of oxidative mitochondrial metabolism in epithelial cancer cells in situ: Visualizing the therapeutic effects of metformin in tumor tissue. Cell Cycle 10(23): 4047-4064.

37. Stincone A, Prigione A, Cramer T, Wamelink MM, Campbell K, et al. (2015) The return of metabolism: Biochemistry and physiology of the pentose phosphate pathway. Biol Rev Camb Philos Soc 90(3): 927-963.

38. Ju HQ, Lu YX, Wu QN, Liu J, Zeng ZL, et al. (2017) Disrupting G6PDmediated Redox homeostasis enhances chemosensitivity in colorectal cancer. Oncogene 36(45): 6282-6292.

39. Yang CA, Huang HY, Lin CL, Chang JG (2018) G6PD as a predictive marker for glioma risk, prognosis and chemosensitivity. J Neurooncol 139(3): 661-670.

40. Chen X, Xu Z, Zhu Z, Chen A, Fu G, et al. (2018) Modulation of G6PD affects bladder cancer via ROS accumulation and the AKT pathway in vitro. Int J Oncol 53(4):1703-1712.

41. Fang Z, Jiang C, Feng Y, Chen R, Lin X, et al. (2016) Effects of G6PD activity inhibition on the viability, ROS generation and mechanical properties of cervical cancer cells. Biochim Biophys Acta 1863(9): 2245-2254.

42. Ohl F, Jung M, Radonic A, Sachs M, Loening SA, et al. (2006) Identification and validation of suitable endogenous reference genes for gene expression studies of human bladder cancer. J Urol 175(5): 1915-1920.

43. Dong T, Kang X, Liu Z, Zhao S, Ma W, et al. (2018) Altered glycometabolism affects both clinical features and prognosis of triple-negative and neoadjuvant chemotherapy-treated breast cancer. Tumour Biol 37(6): 8159-8168.

44. Frederiks WM, Vizan P, Bosch KS, Vreeling SH, Boren J, et al. (2008) Elevated activity of the oxidative and non-oxidative pentose phosphate pathway in (pre)neoplastic lesions in rat liver. Int J Exp Pathol 89(4): 232-240.

45. Ho HY, Cheng ML, Lu FJ, Chou YH, Stern A, et al. (2000) Enhanced oxidative stress and accelerated cellular senescence in glucose-6phosphate dehydrogenase (G6PD)-deficient human fibroblasts. Free Radic Biol Med 29(2): 156-169.

46. Kuo W, Lin J, Tang TK(2000) Human glucose-6-phosphate dehydrogenase (G6PD) gene transforms NIH 3T3 cells and induces tumors in nude mice. Int J Cancer 85(6): 857-864.

47. Debeb BG, Lacerda L, Larson R, Wolfe AR, Krishnamurthy S, et al. (2016) Histone deacetylase inhibitor-induced cancer stem cells exhibit high pentose phosphate pathway metabolism. Oncotarget 7(19): 2832928339.

48. Ma X, Wang L, Huang, Li Y, Yang D, et al. (2017) Polo-like kinase 1 coordinates biosynthesis during cell cycle progression by directly activating pentose phosphate pathway. Nat Commun 8(1): 1506.

49. Zhang HS, Zhang ZG, Du GY, Sun HL, Liu HY, et al. (2019) Nrf2 promotes breast cancer cell migration via up-regulation of G6PD/HIF-1alpha/ Notch1 axis. J Cell Mol Med 23(5): 3451-3463.

50. Ye H, Huang H, Cao F, Chen M, Zheng X, Zhan R (2016) HSPB1 enhances SIRT2-mediated G6PD activation and promotes glioma cell proliferation. PLoS One 11(10): e0164285.

51. Zhang R, Tao F, Ruan S, Hu M, Hu Y, et al. (2019) The TGFbeta1-FOXM1HMGA1-TGFbeta1 positive feedback loop increases the cisplatin resistance of non-small cell lung cancer by inducing G6PD expression. Am J Transl Res 11(11): 6860-6876.

52. Elgendy M, Fusco JP, Segura V, Lozano MD, Minucci S, et al. (2019) Identification of mutations associated with acquired resistance to sunitinib in renal cell cancer. Int J Cancer 145(7):1991-2001.

53. Wang Z, Liang S, Lian X, Liu L, Zhao S, et al. (2015) Identification of proteins responsible for adriamycin resistance in breast cancer cells using proteomics analysis. Sci Rep 5: 9301. 
54. Hong W, Cai P, Xu C, Cao D, Yu W, et al. (2018) Inhibition of glucose-6phosphate dehydrogenase reverses cisplatin resistance in lung cancer cells via the redox system. Front Pharmacol 9: 43.

55. Yin X, Tang B, Li JH, Wang Y, Zhang L, et al. (2017) ID1 promotes hepatocellular carcinoma proliferation and confers chemoresistance to oxaliplatin by activating pentose phosphate pathway. J Exp Clin Cancer Res 36(1):166.

56. Yang X, Ye H, He M, Zhou X, Sun N, et al. (2018) LncRNA PDIA3P interacts with c-Myc to regulate cell proliferation via induction of pentose phosphate pathway in multiple myeloma. Biochem Biophys Res Commun 498(1): 207-213.

57. Shin DH, Park JH, Lee JY, Won HY, Jang KS, et al. (2015) Overexpression of Id1 in transgenic mice promotes mammary basal stem cell activity and breast tumorigenesis. Oncotarget 6(19):17276-17290.

58. Sun Y, Gu X, Zhang E, Park MA, Pereira AM, et al. (2014) Estradiol promotes pentose phosphate pathway addiction and cell survival via reactivation of Akt in mTORC1 hyperactive cells. Cell Death Dis 5: e1231.

59. Hothersall JS, Gordge M, Noronha-Dutra AA (1998) Inhibition of NADPH supply by 6-aminonicotinamide: Effect on glutathione, nitric oxide and superoxide in J774 cells. FEBS Lett 434(1-2): 97-100.

60. Parkhitko AA, Priolo C, Coloff JL, Yun J, Wu JJ, et al. (2014) Autophagydependent metabolic reprogramming sensitizes TSC2-deficient cells to the antimetabolite 6-aminonicotinamide. Mol Cancer Res 12(1): 48-57.

61. Liu CL, Hsu YC, Lee JJ, Chen MJ, Lin CH, et al. (2020) Targeting the pentose phosphate pathway increases reactive oxygen species and induces apoptosis in thyroid cancer cells. Mol Cell Endocrinol 499: 110595.

62. Sharma PK, Varshney R (2012) 2-Deoxy-D-glucose and 6-aminonicotinamide-mediated Nrf2 down regulation leads to radiosensitization of malignant cells via abrogation of GSH-mediated defense. Free Radic Res 46(12):1446-1457.

63. Budihardjo II, Boerner SA, Eckdahl S, Svingen PA, Rios R, et al. (2000) Effect of 6-aminonicotinamide and other protein synthesis inhibitors on formation of platinum-DNA adducts and cisplatin sensitivity. Mol Pharmacol 57(3): 529-538.

64. Zhelev Z, Ivanova D, Bakalova R, Aoki I, Higashi T (2016) Inhibition of the pentose-phosphate pathway selectively sensitizes leukemia lymphocytes to chemotherapeutics by ros-independent mechanism. Anticancer Res 36(11): 6011-6020.

65. Zhao M, Ye M, Zhou J, Zhu X (2019) Prognostic values of transketolase family genes in ovarian cancer. Oncol Lett 18(5): 4845-4857.

66. Ahopelto K, Bockelman C, Hagstrom J, Koskensalo S, Haglund C (2016) Transketolase-like protein 1 expression predicts poor prognosis in colorectal cancer. Cancer Biol Ther 17(2):163-168.

67. Qin Z, Xiang C, Zhong F, Liu Y, Dong Q, et al. (2019) Transketolase (TKT) activity and nuclear localization promote hepatocellular carcinoma in a metabolic and a non-metabolic manner. J Exp Clin Cancer Res 38(1):154.

68. Jayachandran A, Lo PH, Chueh AC, Prithviraj P, Molania R, et al. (2016) Transketolase-like 1 ectopic expression is associated with DNA hypomethylation and induces the warburg effect in melanoma cells. BMC Cancer 16:134.

69. Li J, Zhu SC, Li SG, Zhao Y, Xu JR, et al. (2015) TKTL1 promotes cell proliferation and metastasis in esophageal squamous cell carcinoma. Biomed Pharmacother 74: 71-76.

70. Chao YK, Peng TL, Chuang WY, Yeh CJ, Li YL, et al. (2016) Transketolase serves a poor prognosticator in esophageal cancer by promoting cell invasion via epithelial-mesenchymal transition. J Cancer 7(13): 18041811.

71. Yang H, Wu XL, Wu KH, Zhang R, Ju LL, et al. (2016) MicroRNA-497 regulates cisplatin chemosensitivity of cervical cancer by targeting transketolase. Am J Cancer Res 6(11): 2690-2699.
72. Lu H, Zhu H (2017) Effect of siRNA-mediated gene silencing of transketolase on A549 lung cancer cells. Oncol Lett 14(5): 5906-5912.

73. Wang J, Zhang X, Ma D, Lee WP, Xiao J, et al. (2013) Inhibition of transketolase by oxythiamine altered dynamics of protein signals in pancreatic cancer cells. Exp Hematol Oncol 2:18.

74. Zheng X, Li H (2018) TKTL1 modulates the response of paclitaxelresistant human ovarian cancer cells to paclitaxel. Biochem Biophys Res Commun 503(2): 572-579.

75. Heller S, Maurer GD, Wanka C, Hofmann U, Luger AL, et al. (2018) Gene suppression of transketolase-like protein 1 (TKTL1) sensitizes glioma cells to hypoxia and ionizing radiation. Int J Mol Sci 19(8).

76. Penkowa M, Giralt M, Camats J, Hidalgo J (2002) Metallothionein $1+2$ protect the CNS during neuroglial degeneration induced by 6-aminonicotinamide. J Comp Neurol 444(2):174-189.

77. Shanware NP, Mullen AR, DeBerardinis RJ, Abraham RT (2011) Glutamine: pleiotropic roles in tumor growth and stress resistance. J Mol Med 89(3): 229-236.

78. Yin C, Qie S, Sang N (2012) Carbon source metabolism and its regulation in cancer cells. Crit Rev Eukaryot Gene Expr 22(1): 17-35.

79. Vander HMG, Locasale JW, Swanson KD, Sharfi H, Heffron GJ, et al. (2010) Evidence for an alternative glycolytic pathway in rapidly proliferating cells. Science 329(5998): 1492-1499.

80. Marin-Valencia I, Yang C, Mashimo T, Cho S, Baek H, et al. (2012) Analysis of tumor metabolism reveals mitochondrial glucose oxidation in genetically diverse human glioblastomas in the mouse brain in vivo. Cell Metab 15(6): 827-837.

81. Flavin R, Peluso S, Nguyen P, Loda M (2010) Fatty acid synthase as a potential therapeutic target in cancer. Future Oncol 6(4): 551-562.

82. Santolla MF, Lappano R, De Marco P, Pupo M, Vivacqua A, et al. (2012) $G$ protein-coupled estrogen receptor mediates the up-regulation of fatty acid synthase induced by $17 \beta$-estradiol in cancer cells and cancerassociated fibroblasts. J Biol Chem 287(52): 43234-43245.

83. Zheng J (2012) Energy metabolism of cancer: glycolysis versus oxidative phosphorylation (review). Oncol Lett 4(6): 1151-1157.

84. De Moura MB, Vincent G, Fayewicz SL, Bateman NW, Hood BL, et al. (2012) Mitochondrial respiration-an important therapeutic target in melanoma. PLoS ONE 7(8): e40690.

85. De Oliveira MF, Amoêdo ND, Rumjanek FD (2012) Energy and redox homeostasis in tumor cells. Int J Cell Biol, pp. 1-15.

86. Smolková K, Plecitá-Hlavatá L, Bellance N, Benard G, Rossignol R, et al. (2011) Waves of gene regulation suppress and then restore oxidative phosphorylation in cancer cells. Int J Biochem Cell Biol 43(7): 950-968.

87. Shoshan MC (2012) 3-bromopyruvate: Targets and outcome. J Bioenerg Biomembr 44(1): 7-15.

88. McCubrey JA, Steelman LS, Chappell WH, Abram SL, Franklin RA, et al. (2012) Ras/Raf/MEK/ERK and PI3K/PTEN/Akt/mTOR cascade inhibitors: How mutations can result in therapy resistance and how to overcome resistance. Oncotarget 3 (10): 1068-1111.

89. Martelli AM, Chiarini F, Evangelisti C, Cappellini A, Buontempo F, et al. (2012) Two hits are better than one: Targeting both phosphatidylinositol 3-kinase and mammalian target of rapamycin as a therapeutic strategy for acute leukemia treatment. Oncotarget 3 (4): 371-394.

90. Ko YH, Verhoeven HA, Lee MJ, Corbin DJ, Vogl TJ, et al. (2012) A translational study "case report" on the small molecule "energy blocker" 3-bromopyruvate (3BP) as a potent anticancer agent: From bench side to bedside. J Bioenerg Biomembr 44(1): 163-170.

91. Liapi E, Geschwind JFH (2010) Intra-arterial therapies for hepatocellular carcinoma: Where do we stand? Ann Surg Oncol 17 (5): 1234-1246. 
92. Choi YH, Chung JW, Son KR, So YH, Kim W, et al. (2011) Novel intraarterial therapy for liver cancer using ethylbromopyruvate dissolved in an iodized oil. Acad Radiol 18 (4): 471-478.

93. May BJ, Murthy R, Madoff DC (2012) What's new in trans arterial therapies for hepatocellular carcinoma? Gastrointest Cancer Res 5 (Suppl 1): S14-S19.

94. Ganapathy-Kanniappan S, Kunjithapatham R, Geschwind JF (2012) Glyceraldehyde-3-phosphate dehydrogenase: A promising target for molecular therapy in hepatocellular carcinoma. Oncotarget 3 (9): 940 953.

95. Ganapathy-Kanniappan S, Kunjithapatham R, Geschwind JF (2013) Anticancer efficacy of the metabolic blocker 3-bromopyruvate: specific molecular targeting. Anticaner Res 33 (1): 13-20.

96. Fan QW, Knight ZA, Goldenberg DD, Yu W, Mostov KE, et al. (2006) A dual PI3 kinase/mTOR inhibitor reveals emergent efficacy in glioma. Cancer Cell 9(5): 341-349. 